Meta

Journal des traducteurs

Translators' Journal

\title{
De la traduction comme nomadisme intellectuel : entretien avec Kenneth White, avec la participation de Marie-Claude White
}

\section{Gilles Farcet}

Volume 31, numéro 3, septembre 1986

Prismes de traductions littéraires

Facets of Literary Translation

URI : https://id.erudit.org/iderudit/002720ar

DOI : https://doi.org/10.7202/002720ar

Aller au sommaire du numéro

Éditeur(s)

Les Presses de l'Université de Montréal

ISSN

0026-0452 (imprimé)

1492-1421 (numérique)

Découvrir la revue

Citer cet article

Farcet, G. (1986). De la traduction comme nomadisme intellectuel : entretien avec Kenneth White, avec la participation de Marie-Claude White. Meta, 31(3), 321-331. https://doi.org/10.7202/002720ar d'utilisation que vous pouvez consulter en ligne. 


\title{
DE LA TRADUCTION COMME NOMADISME INTELLECTUEL : ENTRETIEN AVEC KENNETH WHITE, AVEC LA PARTICIPATION DE MARIE- CLAUDE WHITE
}

GILLES FARCET

POETE, TRADUCTEUR, ANGLES SUR L'ANGLIN, FRANCE

\begin{abstract}
C'est à Trébeurden, sur les Côtes du Nord, que vit et travaille Kenneth White. Sa demeure se nomme Gwenved, " monde blanc ", " terme très chargé dans l'ancienne culture celte " selon l'auteur de l'Ode fragmentée à la Bretagne blanche. Écrivain d'origine écossaise établi en France depuis près de vingt ans, titulaire d'une chaire à Paris IV (poétique du $\mathrm{XX}^{\mathrm{e}}$ siècle), Kenneth White produit des livres inclassables à l'intérieur desquels se croisent bien des langues et bien des cultures. La Route bleue, publié chez Grasset en 1983, lui a valu le prix Medicis étranger.

En pénétrant dans son espace de travail, j’éprouve immédiatement la sensation d'évoluer au sein d'une atmosphère dynamique, pluriculturelle, où l'Asie, l'Amérique et l'Europe se rencontrent sans cesse en un dialogue des plus fertiles, favorable à l'éclosion de cette pensée "postmoderne " ébauchée dans son dernier essai (Une apocalypse tranquille, Grasset, 1985). Des livres, de nombreux livres, ceux dans lesquels Kenneth White puise actuellement sa nourriture, sont disposés à même le sol. En haut, l'écrivain laisse reposer un certain nombre de manuscrits "à des stades divers "...

La porte vitrée de la bibliothèque donne sur le jardin. Installés devant des bols de thé chinois, nous commençons à deviser en compagnie de Marie-Claude White, excellente traductrice qui se trouve être l'épouse de l'auteur du Grand rivage.
\end{abstract}

Gilles Farcet : Nous aborderons tout à l'heure les " travaux pratiques " en examinant de près certains passages de vos écrits, mais il me paraît utile d'ouvrir notre dialogue sur une perspective plus vaste. Pour vous, en effet, la traduction n'est pas seulement un exercice intellectuel mais constitue un mode de vie, une manière d'être et de créer à l'intérieur d'un espace ouvert et interculturel...

Kenneth White : Il est effectivement possible de parler de ma démarche en termes de traduction ou d'extrapolation. Je m'intéresse à des cultures autres que celle dans laquelle je suis né car j'estime que toute culture possède ses creux, ses lacunes qu'il importe de combler en allant vers d'autres traditions. Cette extrapolation n'est évidemment possible qu'à partir d'un certain niveau : aucune communication ne pourra s'instaurer entre, par exemple, un paysan français et un paysan hindou, à moins qu'ils ne parlent exclusivement de la terre. Sur le plan idéologique, intellectuel, un abîme les sépare. Par contre, entre un bouddhiste parvenu au terme du bouddhisme et même un catholique tel Maître Eckhart, arrivé au bout de son catholicisme, un dialogue est envisageable. En ce qui me concerne, je me suis donc tourné vers d'autres cultures, notamment la tradition orientale, mais j'ai également tenu à explorer d'anciens courants européens, grecs en particulier. C'est là tout le travail de Heidegger et je m'inscris volontiers 
dans cette mouvance. Ce jeu de l'extrapolation, de l'interculturalisme, délivre en quelque sorte de toutes les fixations, de tous les monolithismes.

G.F. : Afin de prendre du recul vis-à-vis de votre culture d'origine, vous êtes très vite parti : né en Écosse, vous avez, après Glasgow, étudié à Munich, puis à Paris. Par la suite, vous avez voyagé en Orient et en Amérique du Nord ; ces pérégrinations ont d'ailleurs donné lieu à plusieurs livres que vous placez vous-même sous la rubrique "voyages-voyances $"$...

K.W. : Mon premier propos n'était pas de voyager. Mes voyages se sont longtemps bornés à la traversée de la Manche. Je n'éprouvais pas tant le besoin de sortir de la culture britannique, que je ne possédais pas entièrement, que de m'extraire d'un certain état de choses culturel. Donc, dans un premier temps, il ne me venait pas à l'idée de me rendre en Orient, ni même d'aller en Amérique, tout américaniste fou que j'étais. N'espérant pas trouver Walt Whitman dans les rues de New York ou Bouddha dans les rues de Hong Kong, je suis resté entre quatre murs dans des chambres de bonne à Paris où j'étudiais le bouddhisme zen... Tandis que tout le monde se précipitait à Katmandou, je me trouvais à Paris, en train de lire les Upanishads ! Ce n'est que par la suite que je me suis dit " pourquoi pas? » et ai finalement fait le voyage. Une fois parvenu au terme d'un cheminement long, difficile et abstrait, on en arrive à accepter tout le "samsara "du monde et on peut alors se rendre... à Katmandou, à Hong Kong, à Tokyo...

G.F. : Â cette époque, vous avez donc beaucoup voyagé au fil des traductions...

K.W. : Exactement. J'ai toujours été un fervent partisan de la littérature mondiale, convaincu qu'il est désormais impossible de penser ou d'écrire en termes de littérature nationale. Ici, je me réfère à Goethe qui, dès la fin du dix-huitième siècle, affirmait la nécessité de travailler à la littérature mondiale; et ce travail exige le recours à des traductions. Ou sinon, on consacre son temps à l'apprentissage des langues; or, ne faire que cela, c'est peut-être laisser échapper l'essentiel. À l'époque napoléonienne, un linguiste possédant une trentaine de langues a eu un entretien avec l'empereur. Le linguiste ayant pris congé, Napoléon se tourne vers ses conseillers et leur dit : "Voilà un imbécile en trente-cinq langues!" Je n'ai donc pas voulu consacrer mon temps à apprendre une foule de langues; $j$ 'en pratique correctement trois. Les traductions jouent donc un rôle très important. Or, nous avons à cet égard une chance extraordinaire, dans la mesure où la quasi-totalité des textes nous sont actuellement accessibles en traductions. Je m'émerveille toujours à la pensée que Rimbaud ne disposait pas de la matière qui s'offre à nous aujourd'hui. Nous vivons probablement le premier moment de l'histoire où quiconque en éprouvant le désir peut accéder à pratiquement tous les textes de l'humanité ! Quel gâchis si, disposant de ce champ, nous demeurons confinés à l'intérieur de nos limites, occupés à écrire de petites histoires de personnes dans une petite société donnée! C'est un crime contre l'esprit.

G.F. : Dans les notes de Terre de diamant (Grasset, 1983), j'ai relevé le passage suivant : "Quant aux langues, j'ai parfois l'impression qu'elles remuent dans ma tête comme des anguilles. Voici de l'anglais, voici du français et voilà, sous roche, de l'écossais. Et puis, pour changer de famille linguistique et de métaphore, parfois il y a comme un coup de gong dans ma tête, et c'est un idéogramme chinois." (p. 253)

K.W. : Les trois langues que je maitrise à peu près sont bien sûr le français, l'anglais et l'allemand, mais je dispose des instruments de travail nécessaires à l'étude d'autres langues : je possède par exemple des grammaires et des dictionnaires de chinois car cela m'intéresse tout de même d'acquérir une idée de la logique d'une langue. J'ai voulu savoir ce qu'était un idéogramme sans pour autant me préoccuper d'apprendre le chinois. 
G.F. : Le mode d'être et de pensée véhiculé par la langue vous intéresse davantage que la langue elle-même...

K.W. : Exactement. J'ai d'ailleurs fait la même chose avec d'autres langues : le sanscrit, mais également des langues du nord telles que le norvégien, le vieil islandais...

G.F. : Depuis près de vingt ans, vous vivez et travaillez en une langue, le français, qui pour vous demeure malgré tout une langue seconde...

K.W. : Oui, mais je dois d'abord préciser que quatre-vingts pour cent, sinon quatre-vingt-dix pour cent de mes écrits voient d'abord le jour en anglais. Le français est donc effectivement devenu la langue de tous les jours, mais l'anglais est demeuré la langue de l'écriture, sauf exceptions : lorsque je travaille à des articles ou à des essais, j'éprouve un très grand plaisir à manier le français. En fait, je manie le français mais je vis l'anglais, de sorte que pour les textes où prime la sensibilité, l'anglais s'impose de luimême. Beckett a pu se convertir totalement au français dans la mesure où il produit une littérature de l'absurde, extrêmement logique, pour laquelle cette langue convient parfaitement. Par un certain puritanisme, il voulait se débarrasser de tout ce qui était de l'ordre de la sensation; je me trouve dans la situation inverse et suis donc d'autant plus dépendant de la traduction.

G.F. : Ầ propos de l'anglais, vous avez, à l'occasion d'un article, fait la remarque suivante : "Mon anglais, ma langue de base et mon outil d'exploration risque-t-il, du fait que je vis à l'étranger, dans un bain linguistique différent, de se détériorer ? je ne le pense pas, au contraire. Le fait de me mouvoir entre deux langues me conduit à leur prêter plus d'attention."

K.W. : J'ai effectivement pu le constater. Tout le monde s'accorde à dire que vivre à l'étranger constitue un très grand risque pour un écrivain. Ce serait concevable si je faisais des romans réalistes, car il me faudrait me tenir au courant du parler quotidien dans les rues de Londres aujourd'hui, mais ce n'est pas mon cas. Je continue à écrire constamment en anglais et, franchement, je ne ressens aucune perte mais au contraire une sensibilité accrue.

G.F. : Être un écrivain de langue anglaise publiant en France vous conduit à vous interroger sur votre identité ; j'ai relevé à cet égard un passage significatif, toujours dans les notes de Terre de diamant (p. 252) : "Suis-je un poète anglais ou un poète français ? Autrefois, quand on me posait cette question, je répondais, en riant : 'Je suis un poète scotocosmologique !' À présent, sachant trop ce qui se cache sous ce besoin de compartimentation, je me contente de sourire."

K.W. : Cela me fait effectivement sourire beaucoup car je connais des collègues français qui sont tout à fait prêts à dire que White est un très bon poète ... écossais ! Ce besoin de cloisonnement existe parce que chacun s'accroche à sa petite identité ; pour ma part, ce qui m'intéresse, c'est une autre identité, un autre espace littéraire.

G.F. : Et cet autre espace, qui reste à définir, jaillira donc de croisements, il sera le firuit d'une culture polymorphe... Dans Une apocalypse tranquille, vous suivez la piste des Upanishads, de traduction en traduction : une version perse datant du XVII siècle est découverte vers la fin du XVIII ${ }^{\mathrm{e}}$ par le Français Anquetil Duperron qui en donne une traduction latine, laquelle influencera profondément les romantiques allemands. De l'Allemagne à l'Angleterre (via Coleridge et Carlyle), les Upanishads, ainsi d'ailleurs que d'autres écritures orientales, passent aux États-Unis et contribuent à ce que l'on a appelé la « Renaissance américaine ». On les retrouve ensuite chez Yeats, en Irlande... Et vous concluez : "...voilà ce que peut faire une traduction : relier des cultures étrangères l'une à l'autre, et préparer le terrain d'un tertium quid, un nouveau monde intellectuel» (p. 52). 
K.W. : Un nouveau monde intellectuel, voilà ce qui m'intéresse ; ce vers quoi les États-Unis allaient à un moment donné, avec les grands transcendantalistes, mais qui a malheureusement avorté. Par conséquent, le Nouveau Monde reste à découvrir. J'éprouve d'ailleurs beaucoup de réticences à nommer cet autre espace vers lequel je tends. Quelquefois, j'utilise volontairement des termes géographiques : l'Asie, le Labrador, le « chemin du nord profond "... Ce ne sont bien sûr que des métaphores, car ce tertium quid demeure difficile à cerner... Mais je ne verse jamais dans le symbolisme ou dans l'allégorie ; pour moi, la pensée la plus absolue ne doit jamais perdre le contact avec la réalité la plus brute. C'est la raison pour laquelle plutôt que de refaire le Mont Analogue de Daumal, je parle du Labrador. Comment traduire les choses les plus abstruses, les plus compliquées, en langue de tous les jours ? Voilà un problème de l'ordre de la traduction.

G.F. : C'est, je suppose, dans cette optique que vous citez continuellement les auteurs les plus divers, mêlant sans cesse la parole des autres à vos propres textes ; on vous l'a d'ailleurs souvent reproché...

K.W. : On me le reproche continuellement ! Or, en me livrant à cet exercice, je ne désire ni jouer les professeurs ni suppléer à une quelconque faiblesse de mon moi. Au contraire, bien des écrivains ne pourraient se permettre de citer d'autres auteurs de peur que leurs propres textes ne fassent piètre figure à côté... (Rires) Je suis dur, mais c'est comme ça! Je cherche à faire vibrer un réseau et ne me soucie pas de ma petite originalité, de ma propriété privée. Je suis à la recherche d'un monde, et si quelqu'un d'autre a approché ce monde d'une manière admirable, je vais tout simplement le dire, sans lui emprunter ses idées dans les coulisses pour les accommoder à ma sauce. C'est également une question de topologie mentale : j'aime un paysage abrupt et la citation qui survient comme un rocher dans la mer. Cette démarche se retrouve d'ailleurs dans mon enseignement où j'essaie d'ouvrir un espace que j'appelle "poétique du monde ». Je tiens à ne plus faire de distinction absolue entre l'Orient et l'Occident puisqu'à un certain niveau, cette distinction n'existe pas. Je peux être en train de parler de Ezra Pound, par exemple, puis tout naturellement introduire un poète chinois $d u \mathrm{VII}^{\mathrm{e}}$ siècle, sans que l'on ait pour autant l'impression de faire de la littérature chinoise. Évidemment, lorsque l'on a, comme moi, affaire à beaucoup de choses, le défi consiste à accomplir une synthèse sans tomber dans le syncrétisme. J'espère cependant parvenir à une densité accrue par le biais de ce réseau auquel je faisais allusion tout à l'heure.

G.F. : Vous tenez à vous exprimer en une langue simple et limpide ; dans la préface de Terre de diamant, je relève la remarque suivante : " je crois que la plus haute poésie arrive à se dire dans un langage extrêmement clair et simple » (p. 9)...

K.W. : C'est une des raisons pour lesquelles je tiens à l'anglais en tant que langue fondamentale, car je n'y trouve pas cette division entre la langue littéraire et la langue parlée qui pèse sur le français depuis au moins le XVII siècle. Chez Montaigne, qui est l'un de mes grands auteurs, la division ne s'est pas encore faite. Mais après, les écrivains français pratiquent une langue courtisane (le lai de Versailles) ou un style dans lequel prédomine l'architecture de la phrase. La langue anglaise, par contre, a perdu beaucoup de sa graisse. Cela provient peut-être d'une pratique assidue du commerce et du voyage qui ne favorisent pas l'emploi de phrases et de périphrases et également, bien sûr, d'une certaine rudesse anglo-saxonne très présente chez Chaucer, par exemple.

G.F. : Dans Une apocalypse tranquille, vous remarquez : « ...le maintien d'une littérature de qualité dépend en très grande partie d'un art dont la pratique scrupuleuse est rare à l'extrême, qui est l'art de lire. Or, une des meilleures façons de lire est d'essayer de traduire. " (p. 54) Vous avez vous-même commencé par traduire les textes des autres... 
K.W. : Oui, l'un des premiers livres que j'ai publié était une traduction de poèmes d'André Breton (André Breton, 1969 : Selected Poems, Londres, Cape Editions) ; je me suis ensuite attaqué à l'Ode à Charles Fourier (André Breton, 1969 : Ode to Charles Fourier, Londres, Cape Goliard). Ce n'était pas facile... J'ai également fait, en collaboration avec des Chinois, quelques traductions du chinois. Mais c'est surtout à partir du français et de l'allemand que j'ai pu me mesurer aux textes par la lecture. Et effectivement, lire est une pratique difficile que peu de gens maîtrisent ; la plupart se contentent de faire du patinage sur le texte ou de s'y projeter... Sortir de soi pour aller vers le texte n'est pas chose facile et l'on prend conscience de la difficulté, mais aussi du plaisir lié à cette entreprise lorsqu'on essaye de traduire.

G.F. : Votre premier livre publié en France (1964: En toute candeur, Mercure de France) a été traduit par Pierre Leyris... Quel enseignement avez-vous retiré en tant que jeune auteur de cette collaboration avec un grand traducteur?

K.W. : À l'époque, je n'étais que trop heureux de tout laisser entre les mains de Pierre Leyris et je n'ouvrais donc pas la bouche! (Rires) Par la suite (et je ne voudrais pas que ce que je vais dire maintenant soit ressenti comme une critique à l'égard de Leyris), je me suis rendu compte que le style de Pierre, très élaboré, ne correspondait peutêtre pas tout à fait au mien. En lisant En toute candeur, beaucoup de gens ont eu l'impression d'avoir affaire à un écrivain bien plus âgé que moi.

G.F. : Marie-Claude, comment en êtes-vous venue à traduire les livres de Ken ?

M.-C. W. : Cela a coïncidé avec un certain malaise ressenti par Ken vis-à-vis de certains de ses traducteurs qui avaient quelques difficultés à saisir l'humour, le ton de ses textes. Ce qui me frappait depuis longtemps, c'était cette difficulté à traduire en français des textes écrits dans une langue simple; je pensais à des exemples précis : le Vieil homme et la mer, les Aventures d'Huckleberry Finn, des livres pleins d'une langue pittoresque que l'on a un mal fou à rendre en français. Lorsqu'on sort de la belle écriture classique, on tombe dans le style populiste... Il semble qu'il n'y ait rien entre les deux. Et cela aboutit à des tons un peu faux; dans la traduction des Raisins de la colère, par exemple, les paysans emploient de temps à autre une formule un peu pataude mais n'oublient jamais un "ne " dans les négations, alors que ces personnages de Steinbeck parlent un anglais difficile à qualifier... Par conséquent, cela m'intéressait de rechercher ce ton vif et naturel, tandis que les traducteurs de Ken tendaient toujours vers la belle écriture.

G.F. : C'est donc ainsi que vous êtes en quelque sorte devenue la traductrice officielle de Kenneth White...

M.-C. W. : Oui, car je sens tellement de l'intérieur la moindre petite nuance transmise par Ken à travers son style qu'il m'importe de rendre ces subtilités telles que je les ressens, précisément.

K.W. : Ici, je voudrais intervenir. Le fait que je m'élève parfois contre le "Style " conduit certains à prétendre que je n'ai pas de style; en fait, je travaille tout de même beaucoup l'écriture. Il me semble que je joue de manière très complexe sur les nuances...

M.-C. W. : Ce qui déroute probablement certains lecteurs, c'est la grande variété de tons dans tout ce que tu écris : dans la Route bleue, par exemple, on trouvera des passages poétiques, d'autres plus théoriques; des pages empreintes d'une certaine hauteur de ton peuvent précéder des passages rédigés dans un style tout à fait trivial.

G.F. : Comment travaillez-vous? Ken surveille-t-il vos traductions ?

M.-C. W. : Bien entendu, nous revoyons tout ensemble, mais Ken se montre on ne peut plus souple, tout en demeurant exigeant. D'une part il est la plupart du temps satisfait de mes traductions, d'autre part il me laisse prendre certaines libertés. 
G.F. : Ken a incorporé dans les notes de Terre de diamant une remarque de vous à propos de la traduction : «Traduire, c'est faire de la corde raide. Tiré d'un côté par le texte original, on est tiré de l'autre par la langue de réception et le besoin de faire un texte nouveau qui tienne, qui ait une existence en soi. L'art, c'est de maintenir l'équilibre. » (p. 253) Et Ken lui-même précise un peu plus haut : " ...de temps en temps, dans le passage d'une langue à l'autre, parfois à l'initiative des traducteurs, parfois à la mienne, nous nous offrons le luxe d'une petite variation " (p. 253). En consultant les éditions bilingues de Terre de diamant ou du Grand rivage (1980: Le Nouveau Commerce), par exemple, on est effectivement frappé par ces variations, parfois importantes et toujours très heureuses...

M.-C. W. : Lorsque les modifications sont très importantes, elles ont été faites à l'initiative de Ken, car aucun traducteur ne prendrait de telles libertés. Les choses se sont toujours passées ainsi, avec moi comme avec les autres traducteurs. Cela dit, j'ai parfois envie d'exprimer les choses d'une manière quelque peu différente; j'en parle à Ken, et il lui arrive souvent de trouver cela très bien...

K.W. : J'allais le dire! Si Marie-Claude fait une formidable trouvaille qui me paraît meilleure que le texte original, il m'arrive même de changer l'anglais !

G.F. : Vous n'êtes pas des adeptes de la traduction puriste...

M.-C. W. : Certains nous le reprochent parfois ; on accuse Ken de laisser la bride sur le cou à ses traducteurs... Cela procède de tout un fétichisme du pied de la lettre. On révère tellement le mot... Or, si Ken peut se montrer très exigeant, il n'est aucunement tatillon.

K.W. : L'important, c'est que l'esprit souffle. Si Marie-Claude a envie de changer la lettre et si sa version française insuffle vigueur et force au poème, peu m'importe que cela ne corresponde pas sémantiquement au texte original. Je me souviens d'une histoire que m'avait racontée le traducteur de Faulkner, Maurice-Edgar Coindreau : Coindreau peinait sur un paragraphe, et Faulkner lui a dit : "Tu n'as qu'à le sauter »! Personnellement, je n'irais probablement pas jusque-là, mais enfin...

M.-C. W. : En ce qui me concerne, je cherche autant que possible à rester près du texte anglais. Mais face à un problème, nous donnerons toujours la primauté au rythme, à la respiration du texte, plutôt qu'à la lettre.

G.F. : Nous allons maintenant aborder les travaux pratiques, à partir de quelques poèmes. Je voudrais commencer par le texte intitulé "Alchimie familiale " (Terre de diamant, pp. 32-33).

\section{FAMILY ALCHEMY}

When I think of them all: a dancing rascal

a red-bearded fisherman

a red-flag waver

a red-eyed scholar

a drunken motherfucker...

I take a look in the mirror and $I$ wonder

\begin{abstract}
ALCHIMIE FAMILIALE
Quand je songe à tous ces drôles : ce pêcheur du Nord à la barbe rousse celui qui gagnait sa croûte en dansant celui qui dévorait des bouquins en pagaille celui qui se saoulait au lait de la bonté humaine celui qui agitait un drapeau rouge..
\end{abstract}

je me regarde dans la glace et je m'interroge

M.-C. W. : Je ne suis pas l'auteur de cette traduction. Il faut tout de suite préciser que la première édition de ce livre a été traduite par Philippe Jaworski ; puis en 1983 Ken en a publié une nouvelle édition augmentée presque du double, et j'ai alors traduit 
les nouveaux poèmes. Comme il aurait été extrêmement compliqué d'indiquer précisément qui avait fait quoi, nous nous sommes contentés de mentionner les noms des deux traducteurs.

G.F. : La version française de ce texte est en tout cas intéressante. L'ordre a été inversé, le "drunken motherfucker» est devenu " celui qui se saoulait au lait de la bonté humaine "...

K.W. : En ce qui concerne ce poème, le traducteur n'a aucune responsabilité. Nous travaillions en étroite collaboration et dans ce cas précis, c'est moi qui ai pris l'initiative de m'éloigner de l'anglais. Évidemment, vous avez choisi là un exemple extrême. "Drunken motherfucker "... How the hell can you say that in French ? (Rires) "Se saoulait ", c'est pour l'adjectif "drunken ", " motherfucker » c'est celui qui a des rapports intimes avec sa mère, d'où le « lait de la bonté humaine " si l'on pense à " notre mère l'humanité "... Cela aboutit effectivement à quelque chose d'autre, mais je voulais avant tout qu'en français, la traduction donne un poème. Il s'agit donc d'une variation plutôt que d'une traduction à proprement parler. Je me suis dit que les lecteurs auraient finalement deux poèmes. Voilà, vous m'avez pris en flagrant délit ! (rires) Mais cela se produit tout de même très rarement.

G.F. : Passons maintenant à un autre poème de Terre de diamant intitulé " Café du Midi " (pp. 134-135) :

CAFÉ DU MIDI

"Lo cors a fresc, sotil e gai."

\section{Sitting here}

in the shadow

with literature

I see Mireille

keen beauty

crossing the square

$O$ night

please bring me

to her body bare

\section{CAFÉ DU MIDI}

\author{
Assis là \\ dans l'ombre \\ avec un livre \\ je vois Mireille \\ beauté vive \\ traverser le square \\ Ô nuit \\ donne-moi à lire \\ le poème de son corps
}

Ce qui m'intéresse ici, c'est la dernière strophe.

K.W. : Là encore, je suis responsable de cette traduction.

G.F. : Je la trouve très belle. En anglais, les allitérations, entre autres ("bring me to her body bare "), produisent une densité poétique et phonétique qui, du moins de la manière dont je ressens personnellement ces lignes, se trouve admirablement transposée dans le texte français. L'expression " donne moi à lire le poème de son corps " manifeste, traduit véritablement ce que le texte anglais suggérait, alors qu'une traduction littérale (" conduis-moi à son corps nu ") aurait été très pauvre et peu heureuse... On pourrait ici parler de variation dans la fidélité.

K.W. : J'aime aussi beaucoup l'emploi de la demi-rime " lire " et "corps »...

G.F. : De plus, la version française relie la dernière strophe à la première : « Assis ... avec un livre " et " donne-moi à lire... » Prenons un autre cas avec le poème intitulé en français "Hommage à Sesshu Toyo, en passant" (Terre de diamant, pp. 204-205) :

\section{LITTLE HOMAGE TO SESSHU TOYO \\ HOMMAGE À SESSHU TOYO EN PASSANT}

After years in China
Après des années en Chine 
back on the island at the foot of Mount Tenka in Snow Valley Hermitage

painting de retour dans l'île

au pied du mont Tenka

dans l'Ermitage de la vallée des Neiges

écrire l'essentiel paysage

Ce qui saute évidemment aux yeux, c'est le dernier vers...

K.W. : Là encore, je prends l'entière responsabilité de ce texte. J'avais besoin de retrouver une certaine densité en français, et le participe présent "peignant " ne faisait évidemment pas l'affaire : "écrire l'essentiel paysage", par contre, convenait parfaitement. "Écrire un paysage " est une expression chinoise ; de plus, les deux dernières lignes comportent des rimes discrètes qui confêrent au texte une densité phonétique. Ceci m'amène à une autre remarque à propos de l'anglais et du français : je fais un grand usage en anglais du participe présent ; or, cette forme est difficilement utilisable en français, en partie parce qu'elle "sonne" mal. J'ai souvent, en anglais, utilisé le participe présent d'une manière très précise : je m'en servais pour laisser tomber le pronom, car je n'aimais pas cette idée d'une espèce de pronom impérial qui se promène dans le paysage et qui fait des choses ; je voulais donner l'impression d'une participation non pas impersonnelle mais suprapersonnelle, et le français se prête mal à ce type d'approche. Vous pouvez, en anglais, débuter un poème par : "Walking in the morning light »... On ne sait pas de qui il s'agit, c'est presque du chinois ; en français vous serez presque obligé de dire : « je me promène dans la lumière du matin ", à moins de se permettre quelques petites entorses ou d'utiliser un substantif (" promenade dans la lumière du matin "), mais cela ne donnera pas exactement la même chose que le participe présent. Ces histoires de pronom peuvent donner lieu à des prolongements intéressants dans le domaine de la psychologie : pour un enfant autiste, par exemple, il s'agit de faire la conquête du pronom, et dans un champ plus large, pour bien des gens, le summum consiste à pouvoir dire " je ". Or ce qui m'intéresse en littérature, c'est de parvenir à une telle assurance visà-vis de son pronom, de son moi, qu'il devienne possible de le projeter ailleurs ; nous en revenons donc à cette idée d'un autre espace. Alors que nous sommes en grande partie enfermés dans une littérature du «je», du moi social.

M.C. W. : Cet enfermement dans la littérature du moi explique en grande partie certaines réactions hostiles vis-à-vis de ton utilisation fréquente de citations ; ces reproches partent de l'idée qu'un écrivain doit produire des textes qui sont l'expression de son moi, de son individualité. Or, de nombreux lecteurs t'écrivent pour te dire que la lecture de tel ou tel de tes livres les a stimulés, leur a donné envie d'écrire eux-mêmes, et je me demande si cela n'est pas lié à l'absence dans tes textes d'une individualité écrasante qui s'imposerait au monde par sa pensée monolithique... Ils doivent plutôt se sentir en présence d'une sorte de danse dans laquelle ils peuvent entrer.

K.W. : Un espace ou un monde, plutôt qu'un monument qu'on ne peut qu'admirer.

M.C. W. : Pour revenir à la traduction, je crois que les petits poèmes comportent beaucoup plus de libertés du genre de celles que vous avez relevées que les longs poèmes, dont la plupart n'ont pas encore été publiés en livre.

G.F. : Passons maintenant, pour finir, à des extraits du Grand rivage, pp. 14-15, section III :

ah, the gulls :

baagies bluemaws aulins

badochs

goos scutiallans

coobies and scarts ah, les mouettes :

skrev scravedit strigil

gwelenn penn $d u$ fraoiged 


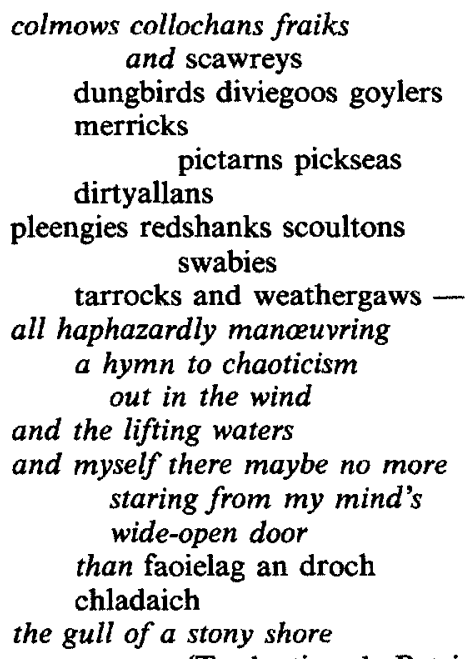

\author{
laboused ar meaz \\ labous aod \\ brini mor koulan
}

$$
\begin{gathered}
\text { labous sant paol } \\
\text { labous sant per } \\
\text { beg melen } \\
\text { gwennili gwenn } \\
\text { gwelan- } \\
\text { volant toutes au hasard } \\
\text { un hymne au chaos } \\
\text { là-dehors dans le vent } \\
\text { et le tumulte des eaux } \\
\text { et moi-même hors de moi } \\
\text { les yeux l'esprit grands } \\
\text { ouverts } \\
\text { rien d'autre que faoielag an } \\
\text { droch chladaich } \\
\text { l'oiseau d'un rivage de pierres }
\end{gathered}
$$

(Traduction de Patrick Guyon et Marie-Claude White)

Dans les notes du Grand rivage, vous expliquez : "Les mots étranges dans la version anglaise sont des noms dialectaux écossais pour toutes sortes de mouettes. Dans la version française, ils sont remplacés par des mots bretons. " (p. 122)

K.W. : Ici, je peux raconter une petite anecdote : ne connaissant pas le breton, j'avais écrit à un spécialiste de cette langue en lui demandant de m'indiquer plusieurs noms de mouettes en breton, étant convaincu qu'il en existait beaucoup. Et il m'a répondu d'une manière un peu cavalière en me donnant le nom générique que je connaissais déjà puisque je possédais un dictionnaire. J'ai alors demandé à quelques amis qui allaient passer leurs vacances en Bretagne de se rendre dans les petits ports et de demander des noms de mouettes. Ils sont donc allés à la pêche aux mots et en sont revenus avec une dizaine ou une douzaine de noms.

G.F. : Pourquoi avez-vous tenu à transposer ces noms de l'écossais au breton ?

K.W. : L'Anglais qui lit ce passage se trouve confronté à une langue étrangère ; de même, il me fallait un corps étranger dans le texte français. Évidemment, le rapport entre l'anglais et l'écossais et entre le français et le breton n'est pas exactement le même, mais comment trouver l'exact équivalent ? Peut-être aurait-il fallu chercher du côté du picard...

G.F. : Pour conclure notre entretien, j'aimerais revenir à Une apocalypse tranquille, votre dernier livre dans lequel vous citez George Steiner, l'auteur d'Après Babel :

Plus que les linguistes, et bien avant eux, poètes et traducteurs ont exploré l'épiderme du parler... Pour découvrir le langage, il faut abandonner les "structures profondes " de la grammaire transformationnelle pour celles plus profondes encore de la poésie...

Et vous commentez : "Des phrases comme celles-là indiquent un changement très significatif dans le climat mental $\gg($ p. 56).

K.W. : Je le pense, en effet. Mais il y a encore beaucoup de travail à faire.

La nuit est tombée lorsque Marie-Claude et Kenneth White me reconduisent à mon hôtel. Avant de m'endormir, je marche le long de la plage, presque à l'aveuglette, afin de m'emplir des sons et des senteurs du "grand rivage». 


\section{NOTES BIOGRAPHIQUES}

Né en 1936 à Glasgow (Écosse), fils d'un signaleur de chemins de fer, Kenneth White a poursuivi des études de littérature et de philosophie à Glasgow, Munich et $\mathrm{Pa}$ ris. Installé en France depuis une vingtaine d'années, il occupe depuis 1983 une chaire (poétique du XXe siècle) à l'Université de Paris-Sorbonne. S'il n'a aucunement rompu avec ses origines celtes (tout en fuyant le régionalisme), ses œuvres principales ont d'abord été publiées en France et c'est jusqu'à présent au sein de l'espace littéraire francophone que son influence se fait le plus nettement sentir.

La place qu'il occupe est toute particulière : Kenneth White, en effet, n'écrit pas de romans et poursuit son travail en dehors des modes et du parisianisme. Ses influences sont nombreuses, fort diverses et souvent inhabituelles. Parmi les plus marquantes, mentionnons la poésie américaine (Whitman, Pound, Williams, Olson) et tous ceux chez lesquels l'espace du nouveau monde se fait sentir (Thoreau, Melville, sans oublier les Amérindiens), la poésie celte, les poètes chinois et japonais (White est un praticien doué du haiku), les surréalistes... N'oublions pas des écrivains tels que Segalen, Cendrars, Delteil, Miller, D.H. Lawrence, Dylan Thomas, McDiarmid, qui ont tous en commun d'être d'extravagantes individualités. White aspire en effet à vaquer en dehors, il travaille à sortir d'une " culture " fatiguée, faite d'inflation verbale ou de petites histoires nombrilistes pour ouvrir un nouvel espace plus dense, plus vigoureux. Son écriture s'articule sur la quête de ce qu'il nomme " le monde blanc", une expérience lumineuse du monde à travers laquelle il réconcilie Orient et Occident.

Nombreux sont aujourd'hui ceux qui se placent dans cette perspective singulièrement vivifiante : sans tapage, les livres de Kenneth White disparaissent des librairies, des dossiers, des expositions, des thèses même, lui sont consacrés et ses cours à la Sorbonne, qu'il ne sépare pas de ses créations, ouvrent de nouvelles voies à des étudiants en quête de "gai savoir".

Il distingue lui-même parmi ses nombreuses publications trois types de livres :

Il existe trois sortes de livres que je trouve utile de pratiquer : les pérégrinations où je m'expose, les recherches où j'explore, les poèmes où je présente des moments plus exacts que les autres. (Terre de diamant, p. 9)

\section{La bibliographie ci-dessous respectera donc sa propre classification.}

Récits, cheminements, voyages-voyances :

Les Limbes incandescents, traduction Patrick Mayoux, Paris, Denoël, collection les Lettres nouvelles, 1976. Dérives, plusieurs traducteurs, Paris, Lettres nouvelles/Maurice Nadeau, 1978.

Lettres de Gourgounel, traduction Gil et Marie Jouanard, Paris, Les Presses d'aujourd'hui, 1979.

L'Écosse avec Kenneth White, Paris, Flammarion, 1980.

Le Visage du vent d'Est, traduction Marie-Claude White, Paris, Les Presses d'aujourd'hui, 1980.

La Route bleue, traduction de Marie-Claude White, Paris, Grasset, 1983.

\section{Poésie}

En toute candeur, édition bilingue, traduction de Pierre Leyris, Paris, Mercure de France, 1964.

Mahamudra, le grand geste, édition bilingue, traduction Marie-Claude White, Paris, Mercure de France, 1979.

Le Grand rivage, édition bilingue, traduction Patrick Guyon et Marie-Claude White, Paris, Le Nouveau commerce, 1980.

Scènes d'un monde flottant, édition bilingue revue et augmentée, traduction Marie-Claude White, Paris, Grasset, 1983.

Terre de diamant, édition bilingue revue et augmentée, traduction Philippe Jaworski, Marie-Claude White et l'auteur, Paris, Grasset, 1983.

Kenneth White a également publié de nombreux poèmes en éditions à tirage limité : 
Autumn at Luk Wu Temple, poèmes, Paris, Orange Export Limited, 1976.

Crow Meditation Text, poème, Villeneuve-lès-Avignon, La Maison du livre et des mots, 1977.

La Résidence de la solitude et de la lumière, poème, édition bilingue, traduction Marie-Claude White, JeanMichel Michelena et l'auteur, aquarelles de R. Christien, dessins de F. Limérat, Bordeaux, William Blake and Co., 1978.

Pierres de la forêt brumeuse, poèmes, sérigraphies de Frédéric Pou, Paris, Ombelle, 1978.

Earling Morning Light on Loch Sunart, poème, Paris, Orange Export Limited, 1979.

Ode fragmentée à la Bretagne blanche, poèmes, sérigraphies d'Ed. Sommer, Bordeaux, William Blake and Co., 1980.

\section{Essais, recherches :}

Segalen, théorie et pratique du voyage, traduction Michelle Tran Van Khai, Paris et Lausanne, Alfred Eibel éditeur, 1979.

La Figure du dehors, Paris, Grasset, 1982. (Le livre essentiel pour entrer dans la démarche de Kenneth White.)

Une apocalypse tranquille, Paris, Grasset, 1985.

\section{Traductions :}

André Breton (1968) : Selected Poems, Londres, Cape Editions.

André Breton (1969) : Ode to Charles Fourier, Londres, Cape Goliard.

Edgar Lee Masters (1976) : Spoon River, avec Michel Pétris, Paris, Champ libre.

Kenneth White a publié, en avril 1986, un nouveau livre de poèmes : Atlantica, édition bilingue, traduction Marie-Claude White, Paris, Grasset. 